\title{
Effects of nicorandil on p120 expression in the spinal cord and dorsal root ganglion of rats with chronic postsurgical pain
}

\author{
SAI-SAI HUANG ${ }^{1}$, SU CAO $^{2}$, CUI E. LU ${ }^{2}$, YI-BIN QIN ${ }^{2}$ and JIAN-PING YANG ${ }^{1}$ \\ ${ }^{1}$ Department of Anesthesiology, The First Affiliated Hospital of Soochow University, Suzhou, Jiangsu 215000; \\ ${ }^{2}$ Department of Anesthesiology, Affiliated Hospital of Nantong University, Nantong, Jiangsu 226001, P.R. China
}

Received February 20, 2020; Accepted August 21, 2020

DOI: $10.3892 / \mathrm{mmr} .2020 .11546$

\begin{abstract}
Chronic postsurgical pain (CPSP) has a high incidence, but the underlying mechanism is not well understood. Accumulating evidence has suggested that central sensitization is the main mechanism of pain. To study the role of p120 in CPSP, a skin/muscle incision and retraction (SMIR) model was established, and immunofluorescence staining and western blotting were performed to analyze the expression of p120 in the spinal cord and dorsal root ganglion (DRG). The results demonstrated that SMIR increased the expression of p120 in the DRG and the spinal cord compared with the naive group. Furthermore, it was demonstrated that p120 was mainly distributed in the glial fibrillary acidic protein-positive astrocytes in the spinal cord, and in the neurofilament 200-positive medium and large neurons in the DRG. Our previous studies have shown that adenosine triphosphate-sensitive potassium channel (KATP) agonists can reduce postoperative pain in rats. Therefore, the changes in p120 were observed in the DRG and spinal cord of rats following the intraperitoneal injection of nicorandil, a KATP agonist. It was demonstrated that nicorandil administration could relieve mechanical pain experienced following SMIR in rats, and decrease the expression of p120 in the DRG and spinal cord. The results revealed that p120 may contribute to the prophylactic analgesic effect of nicorandil, thus providing a novel insight into the mechanism of CPSP prevention.
\end{abstract}

\section{Introduction}

It has been reported that $8-34 \%$ of patients experience chronic postsurgical pain (CPSP) following surgery, which results in a decrease in the quality of postoperative daily life (1). This is a difficult clinical problem. The mechanism underlying the transformation of acute pain to chronic pain following tissue

Correspondence to: Dr Jian-Ping Yang, Department of Anesthesiology, The First Affiliated Hospital of Soochow University, 899 Pinghai Road, Gusu, Suzhou, Jiangsu 215000, P.R. China E-mail: 177368688@qq.com

Key words: nicorandil, p120, chronic postsurgical pain, dorsal root ganglion, spinal cord damage remains to be elucidated. An improved understanding of this mechanism is the key for clinically preventing CPSP.

Growing evidence has indicated that nerve damage causes the resting astrocytes of the spinal cord to enter into an active state, which is necessary for the development and maintenance of pain (2-4). The astrocytes in the central nervous system (CNS) form a highly interconnected network via gap junctions or adherens junctions (5). In some pain models, there is an increase in the number of gap junction channels and a series of junction proteins, such as connexin43 (Cx43) and p120, leading to the secretion of various types of cytokines that are involved in inducing pain (6). The role of $\mathrm{Cx} 43$ in chronic pain has been studied previously (7-9). The purpose of the present study was to explore the role of p120 in the maintenance of CPSP by detecting the expression of p120 in the DRG and spinal cord of rats with CPSP.

Our previous studies have shown that adenosine triphosphate-sensitive potassium channel (KATP) agonists may relieve postoperative pain in rats by inhibiting the apoptosis of vascular endothelial cells $(10,11)$. KATP agonists can inhibit touch-induced pain in a dose-dependent manner (11). Notably, nicorandil is the first clinical KATP opener, which has been reported to exhibit activities in models of inflammatory and nociceptive pain $(12,13)$. The present study attempted to extend the investigation on the antinociceptive activity of nicorandil by evaluating its effects on the expression of p120 and also to evaluate the possible mechanisms involved in the antinociceptive activity of this drug through the p120 pathway.

\section{Materials and methods}

Animals. A total of 42 male Sprague-Dawley rats (weight, 200-250 g; age, 8-10 weeks) were provided water and feed ad libitum until 3 days prior to the experiment. The rats were house with a $12 \mathrm{~h}$ light/dark cycle at $23 \pm 1^{\circ} \mathrm{C}$ and $55-60 \%$ humidity. The rats were provided by the Experimental Animal Center of Nantong University (Nantong, China) and the study procedures were approved by the Animal Care and Use Committee of Nantong University (approval no. 20171015S1051122).

Experimental design and drugs. The rats were randomly divided into seven groups ( $\mathrm{n}=6 /$ group). Naive group, no treatment was performed; sham group, the rats received an incision 
through the skin and muscle; skin/muscle incision and retraction (SMIR) (14) group, the rats underwent 1-h retraction following the skin/muscle incision; SMIR + nicorandil groups, referring to the dose of nicorandil in the relevant literature (15-17), the rats were intraperitoneally injected with either low $(1.0 \mathrm{mg} / \mathrm{kg})$, middle $(1.5 \mathrm{mg} / \mathrm{kg})$ or high dose nicorandil $(2.0 \mathrm{mg} / \mathrm{kg})$ separately $0.5 \mathrm{~h}$ prior to the SMIR procedure; SMIR + nicorandil $(1.5 \mathrm{mg} / \mathrm{kg})+$ glibenclamide $(10.0 \mathrm{mg} / \mathrm{kg})$ group, $0.5 \mathrm{~h}$ before SMIR modeling, a mixture of $1.5 \mathrm{mg} / \mathrm{kg}$ nicorandil (cat. no. N3539; Sigma-Aldrich; Merck KGaA) and $10.0 \mathrm{mg} / \mathrm{kg}$ glibenclamide (cat. no. PHR1287; Sigma-Aldrich; Merck $\mathrm{KGaA}$ ) was injected intraperitoneally.

Behavioral testing. The mechanical withdrawal threshold (MWT) was detected prior to, and 1, 3, 7 and 14 days following SMIR surgery. The rats were habituated to the testing environment for at least $30 \mathrm{~min}$ before testing. Mechanical allodynia was assessed using the Up-Down paradigm (18) with von Frey filaments (IITC Life Science, Inc.) ranging between 1.4 and $26 \mathrm{~g}$. Shrinking, swinging or paw licking were regarded as positive reactions. Each filament was presented five times within $30 \mathrm{sec}$ to determine the response threshold. If the response was not elicited at least twice, the next ascending von Frey filament was applied until at least two responses were observed.

Immunofluorescence staining. On day 7 after SMIR, the rats were anesthetized with isoflurane (induction with 3-4\%, maintenance with 1-2\%) and perfused transcardially with PBS followed by $4 \%$ paraformaldehyde in PBS (250 ml; pH 7.0). After perfusion, the L3-L5 spinal cord and DRG tissues from rats in the SMIR group were extracted and post-fixed in the same fixative at $4^{\circ} \mathrm{C}$ overnight, and then placed in $20 \%$ and subsequently in $30 \%$ sucrose solution at $4^{\circ} \mathrm{C}$ overnight. After freezing sequentially, the tissues were continuously sectioned at $6 \mu \mathrm{m}$ and stored at $-20^{\circ} \mathrm{C}$. These sections were selected randomly and blocked with $5 \%$ serum antibody blocking solution (Beyotime Institute of Biotechnology) for $2 \mathrm{~h}$. The tissue slices were then incubated independently with antibodies against p120 (1:300; Santa Cruz Biotechnology, Inc; cat. no. sc-23873), glial fibrillary acidic protein (GFAP; 1:5,000; EMD Millipore; cat. no. AG230), calcitonin gene-related peptide (CGRP; 1:800; Cell Signaling Technology, Inc.; cat. no. 14959), NeuN (1:5,000; EMD Millipore; cat. no. SAB4300883), ionized calcium-binding adapter molecule 1 (Iba1; 1:800; FUJIFILM Wako Pure Chemical Corporation; cat. no. 019-19741) and isolectin B4 (IB4; 1:1,000; Advanced Targeting Systems, Inc.; cat. no. PR-02) at $4^{\circ} \mathrm{C}$ overnight, then co-incubated with $\mathrm{Cy} 3$ or FITC-conjugated secondary antibodies (1:1,000, Jackson ImmunoResearch Laboratories, Inc.; cat. no. 115-165-205, 115-095-205) in the dark for $2 \mathrm{~h}$ at room temperature. A total of five sections were randomly selected from the spinal cord and DRG of each rat. The localizations of p120 in the spinal cord dorsal horn and DRG were examined under a fluorescence microscope (Olympus Corporation) in the dark to capture images.

Western blotting. The rats were anesthetized and sacrificed as previously described, and the L3-L5 spinal cord and DRG tissues were homogenized in sodium dodecyl sulfate (SDS) sample buffer containing a mixture of protease and phosphatase inhibitors (Sigma-Aldrich; Merck KGaA), and measured with a bicinchoninic acid protein assay kit (Beyotime Institute of Biotechnology). For separation, $30 \mu \mathrm{g}$ total protein per gel lane was loaded onto $10 \%$ gels (Beyotime Institute of Biotechnology). The separated proteins were then transferred onto nitrocellulose membranes. The membranes were incubated for $2 \mathrm{~h}$ at room temperature in tris-buffered saline and Tween-20 blocking solution containing $5 \%$ skimmed milk, followed by overnight incubation at $4{ }^{\circ} \mathrm{C}$ in blocking solution containing primary antibodies against the following proteins: p120 (1:300; Santa Cruz Biotechnology, Inc.; cat. no. sc-23873) and GAPDH (1:5,000; Sigma-Aldrich; Merck KGaA; cat. no. G2267). Membranes were washed three times and incubated with the anti-mouse or anti-rabbit peroxidase-conjugated secondary antibodies (1:2,000; Jackson Immuno Research Laboratories, Inc.; cat. nos. 115-035-003, 111-005-003, respectively) at room temperature for $2 \mathrm{~h}$. After washing, immunolabeling was detected using the Tanon 2500 gel imaging system (Tanon Science and Technology Co., Ltd.) and hypersensitive ECL chemiluminescence detection kit (Absin). ImageJ software (version no. 1.8.0, National Institutes of Health) was used to capture and analyze the intensity of the bands.

Statistical analysis. The data were presented as mean \pm standard error of mean of at least three experimental repeats. Data were analyzed using one- or two-way analysis of variance followed by Bonferroni's or Dunnett's post hoc test. $\mathrm{P}<0.05$ was considered to indicate a statistically significant difference.

\section{Results}

SMIR induces persistent mechanical allodynia and nicorandil attenuates mechanical allodynia. To study the mechanism underlying CPSP, an SMIR model was established according to a previous study (14) (Fig. 1A). As shown in Fig. 1B, the MWT significantly decreased on postsurgical days 1, 3, 7 and 14 in the sham, SMIR +1.5 and SMIR $+2.0 \mathrm{mg} / \mathrm{kg}$ nicorandil groups, as compared with the SMIR group, in a time-dependent manner. Whereas, no statistical difference was observed in the MWT of the SMIR $+1.0 \mathrm{mg} / \mathrm{kg}$ nicorandil group compared with the SMIR group $(\mathrm{P}>0.05)$. It was identified that the anti-nociceptive effects of 1.5 and $2.0-\mathrm{mg} / \mathrm{kg}$ nicorandil were more pronounced compared with $1.0 \mathrm{mg} / \mathrm{kg}$ nicorandil $(\mathrm{P}<0.05)$, and no significant difference was observed between 1.5 and $2.0 \mathrm{mg} / \mathrm{kg}(\mathrm{P}>0.05$; Fig. 1B). These results suggested that 1.5 and $2.0 \mathrm{mg} / \mathrm{kg}$ nicorandil may inhibit SMIR-induced mechanical hyperalgesia, and that $1.5 \mathrm{mg} / \mathrm{kg}$ nicorandil may be the most effective dose. Therefore, $1.5 \mathrm{mg} / \mathrm{kg}$ nicorandil was used to determine whether the anti-nociceptive effects of a KATP activator could be relieved by the KATP blocker glibenclamide $(10.0 \mathrm{mg} / \mathrm{kg})$. As expected, injection of glibenclamide together with nicorandil reversed the anti-mechanical nociceptive effects of nicorandil on postsurgical days 1, 3, 7 and 14. These findings suggested that nicorandil may alleviate CPSP through activating KATP.

Effects of CPSP on expression of p120 in the spinal cord and $D R G$. To explore the role of p120 in central sensitization of CPSP and its possible mechanism, changes in the expression 
A

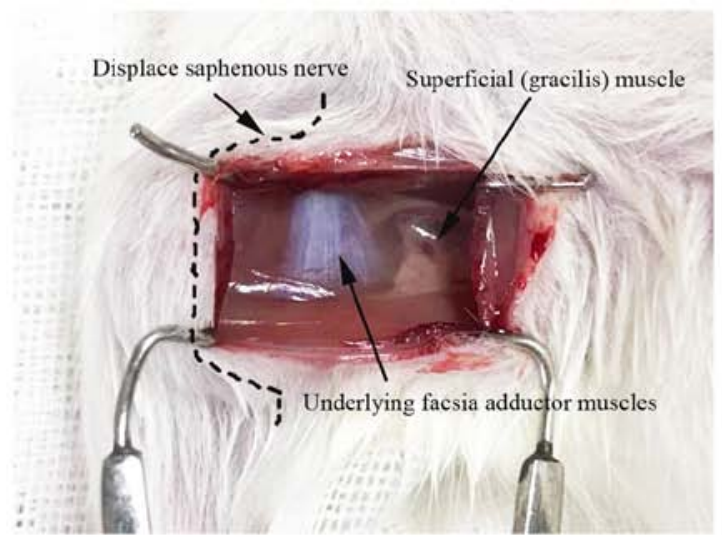

B

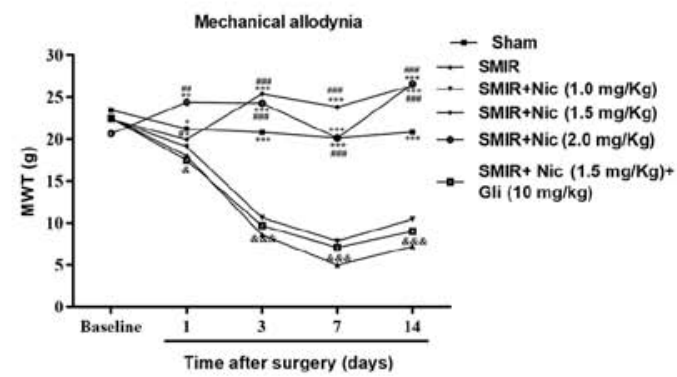

Figure 1. Establishment of the SMIR model in rats and detection of MWT. (A) SMIR model was established; the saphenous nerve was pulled and displaced. (B) Comparison of MWT in the sham, SMIR, Nic-treated groups and SMIR + Nic $(1.5 \mathrm{mg} / \mathrm{kg})+$ Gli group $(\mathrm{n}=6)$. ${ }^{*} \mathrm{P}<0.05,{ }^{* * *} \mathrm{P}<0.01,{ }^{* * * *} \mathrm{P}<0.001 \mathrm{vs}$. SMIR group; ${ }^{\#} \mathrm{P}<0.05,{ }^{\# \#} \mathrm{P}<0.01,{ }^{\# \# \#} \mathrm{P}<0.001$ vs. SMIR + Nic $(1.0 \mathrm{mg} / \mathrm{kg})$ group; ${ }^{\&} \mathrm{P}<0.05$, \&\&\& $\mathrm{P}<0.001 \mathrm{vs}$. SMIR + Nic $(1.5 \mathrm{mg} / \mathrm{kg})$ group. SMIR, skin/muscle incision and retraction; MWT, mechanical withdrawal threshold; Nic, nicorandil; Gli, glibenclamide.
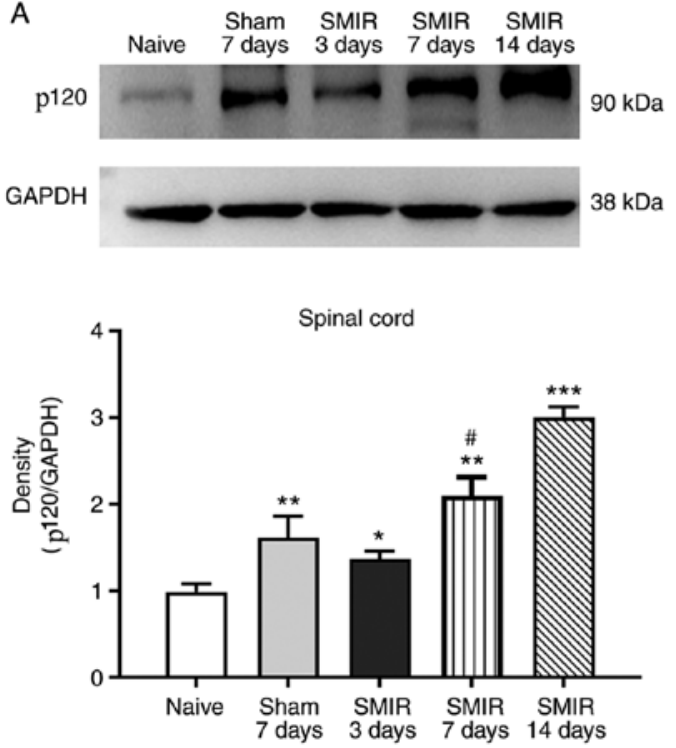
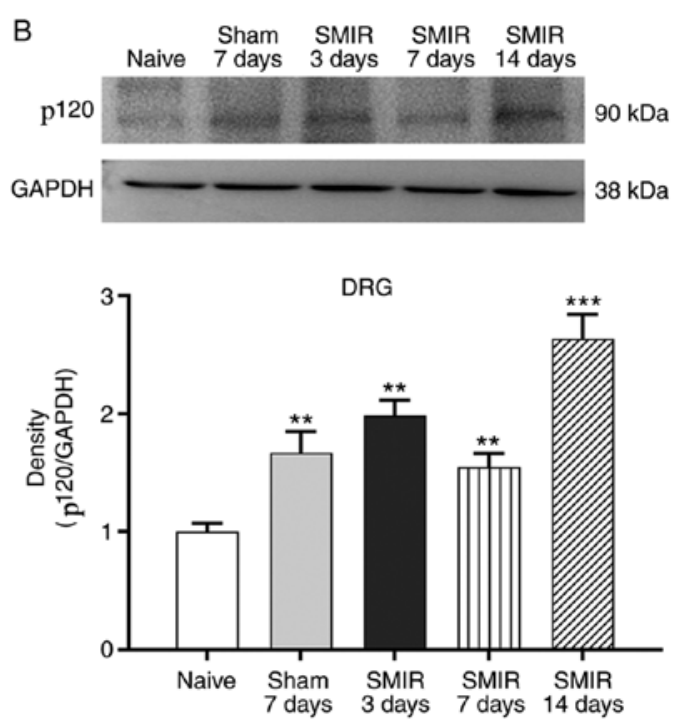

Figure 2. Expression of p120. (A) In the spinal cord, the expression of p120 was significantly higher in the SMIR group compared with in the naive group on postsurgical days 3, 7 and 14. In the sham group, the expression of p120 increased significantly on postsurgical day 7 . Compared with on postsurgical day 7 in the sham group, the SMIR group exhibited significantly increased expression of p120 on postsurgical day 7 . (B) In the DRG, compared with the naive group, SMIR increased p120 expression on postsurgical days 3, 7 and 14. In the sham group, p120 expression increased significantly on postsurgical day 7 . No significant difference was observed between the sham and SMIR groups on postsurgical day $7(\mathrm{P}>0.05)$. ${ }^{*} \mathrm{P}<0.05,{ }^{* *} \mathrm{P}<0.01,{ }^{* * * *} \mathrm{P}<0.001$ vs. naive group; ${ }^{\#} \mathrm{P}<0.05$ vs. sham group. SMIR, skin/muscle incision and retraction; DRG, dorsal root ganglion.

of p120 in the spinal cord and DRG were examined on postsurgical days 3, 7 and 14 in the SMIR and sham groups. It was identified that SMIR-induced CPSP enhanced the expression of p120 in the spinal cord and DRG of rats compared with in the naive group (Fig. 2A and B). There was also a high expression of p120 on postsurgical days 7 and 14 in the sham group (Figs. S1 and S2). As the 7th day after surgery may be the turning point of the transformation from acute pain to chronic pain, the present study selectively compared the expression of p120 on postsurgical day 7 in the sham group with that in the SMIR group. The results demonstrated that the expression of p120 in the spinal cord of the SMIR group was higher compared with that of the sham group on postsurgical day 7, whereas no significant difference was observed in DRG between the two groups (Fig. 2A and B).
Immunofluorescence staining depicts localization of p120 in the spinal cord. To study the location of $\mathrm{p} 120$, spinal cord sections of rats in the SMIR group at postsurgical day 7 were stained with the neuronal marker NeuN, astrocyte marker GFAP and microglia marker Iba1. Results revealed that p120 was distributed in GFAP-positive astrocytes. However, p120-positive staining was not observed in the Iba1- and NeuN-positive neurons (Fig. 3A-C).

Immunofluorescence staining demonstrates the localization of p120 in the DRG. At postsurgical day 7, DRG tissue sections were stained with p120, and the medium and large neuronal marker NF200, or the medium and small neuronal markers CGRP and IB4. The results revealed that p120 was mainly distributed in the NF200-positive medium and large neurons, 
A

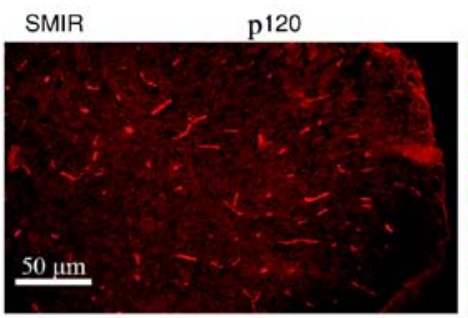

B SMIR

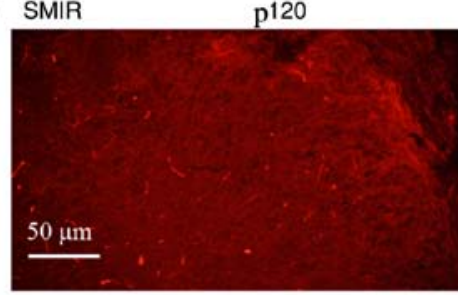

C SMIR

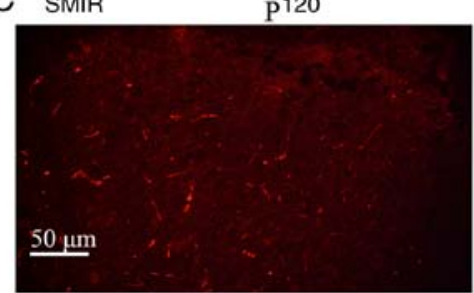

GFAP

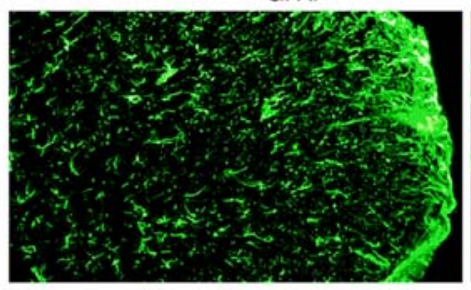

lba1

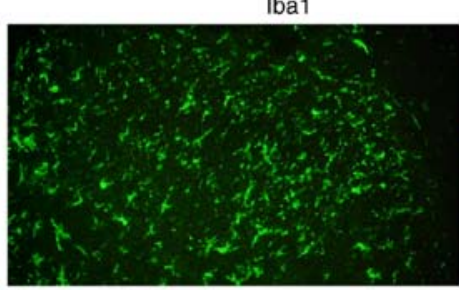

NeuN

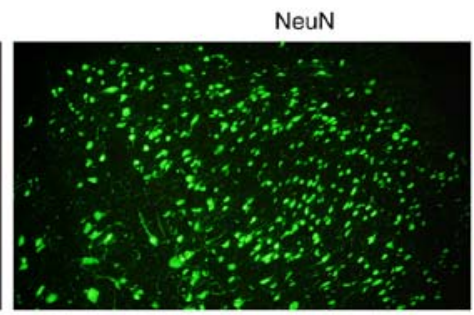

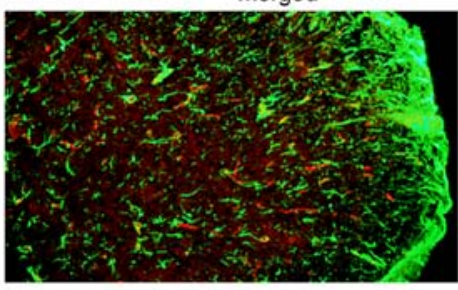

Merged
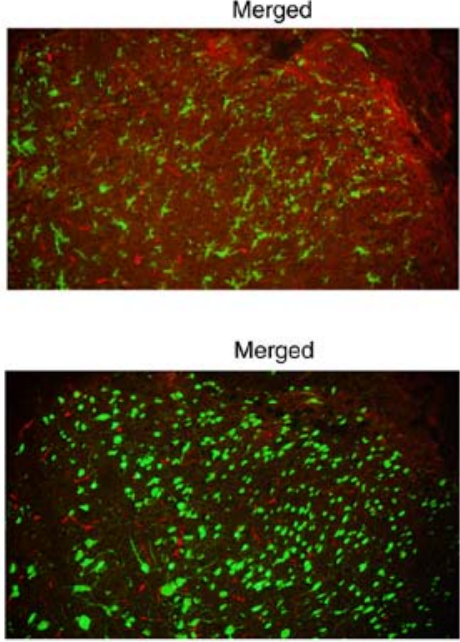

Figure 3. Immunofluorescence staining. Immunofluorescence staining demonstrated that in the spinal cord, p120 (A) colocalizes with the GFAP-positive astrocytes, but does not colocalize with (B) Iba1- or (C) NeuN-positive neurons. GFAP, glial fibrillary acidic protein; Iba1, ionized calcium binding adapter molecule 1; SMIR, skin/muscle incision and retraction.

but not in the CGRP- and IB4-positive medium and small neurons (Fig. 4A-C).

Effect of nicorandil on changes in expression of p120 in the spinal cord and DRG. To study the mechanism underlying the analgesic effect of KATP agonists, the present study detected the changes in the expression of p120 in the spinal cord and DRG following intraperitoneal injection of $1.5 \mathrm{mg} / \mathrm{kg}$ nicorandil $0.5 \mathrm{~h}$ before SMIR. The changes in the expression of p120 were observed on postsurgical day 7 . The results suggested that SMIR promoted the expression of p120 in the spinal cord and DRG, whereas nicorandil treatment decreased the expression of p120 (Fig. 5A and B).

\section{Discussion}

In 2008, Flatters et al (14) developed the SMIR model to study CPSP. In the current study, MWT was significantly decreased following SMIR in a time-dependent manner compared with the sham group, indicating that the pain model was effective. Recently, the prevalence of CPSP has been increasingly regarded as a public health problem; thus, the present study established SMIR, simulating the state of CPSP in rats, to explore the potential mechanism of action of nicorandil in relieving pain.

The astrocytes in the spinal cord are considered to be the key point for central sensitization $(19,20)$, which is the main mechanism of pain induction (21-23). Astrocytes in the CNS form a highly interconnected network via complexes formed by connexins, such as p120 and Cx43 $(5,24)$. These complexes are involved in the regulation of cell adhesion, migration, invasion and proliferation (25). In some pain models, the increased expression of a series of connexins, especially $\mathrm{Cx} 43$, leads to the secretion of various types of cytokines, and participates in the occurrence and development of pain $(26,27)$. A previous study examined the role of $\mathrm{Cx} 43$ in chronic pain (28); however, to the best of our knowledge, the relationship between p120 and chronic pain remains to be elucidated. p120 is known to exhibit a role in adhesion among cells and in signal transduction in cells (29-32). It has also been reported that p120 protects against post-traumatic inflammation by regulating the adherens and tight gap junctions $(33,34)$. When the body is subjected to peripheral stimulation, the DRG first senses this change and activates the DRG neurons, which then transmit to the dorsal horn of the spinal cord, causing abnormal neuronal excitability, which leads to the sensation of pain in the brain (35). Therefore, the present study selectively detected changes in the expression of p120 in the spinal cord and DRG of rats with CPSP, and examined the localization of p120 in the two tissues. Results revealed that SMIR increased the expression of p120 in the DRG and spinal cord compared with in the naive group. Furthermore, p120 was mainly identified to be distributed in GFAP-positive astrocytes in the spinal cord, and in the NF200-positive medium and large neurons in the DRG. The interaction between the astrocytes and neurons may regulate synaptic transmission through p120, which contributes to central sensitization of spinal cord pain signals (36), indicating that p120 may be involved in the maintenance of CPSP through astrocyte-neuron interaction. 

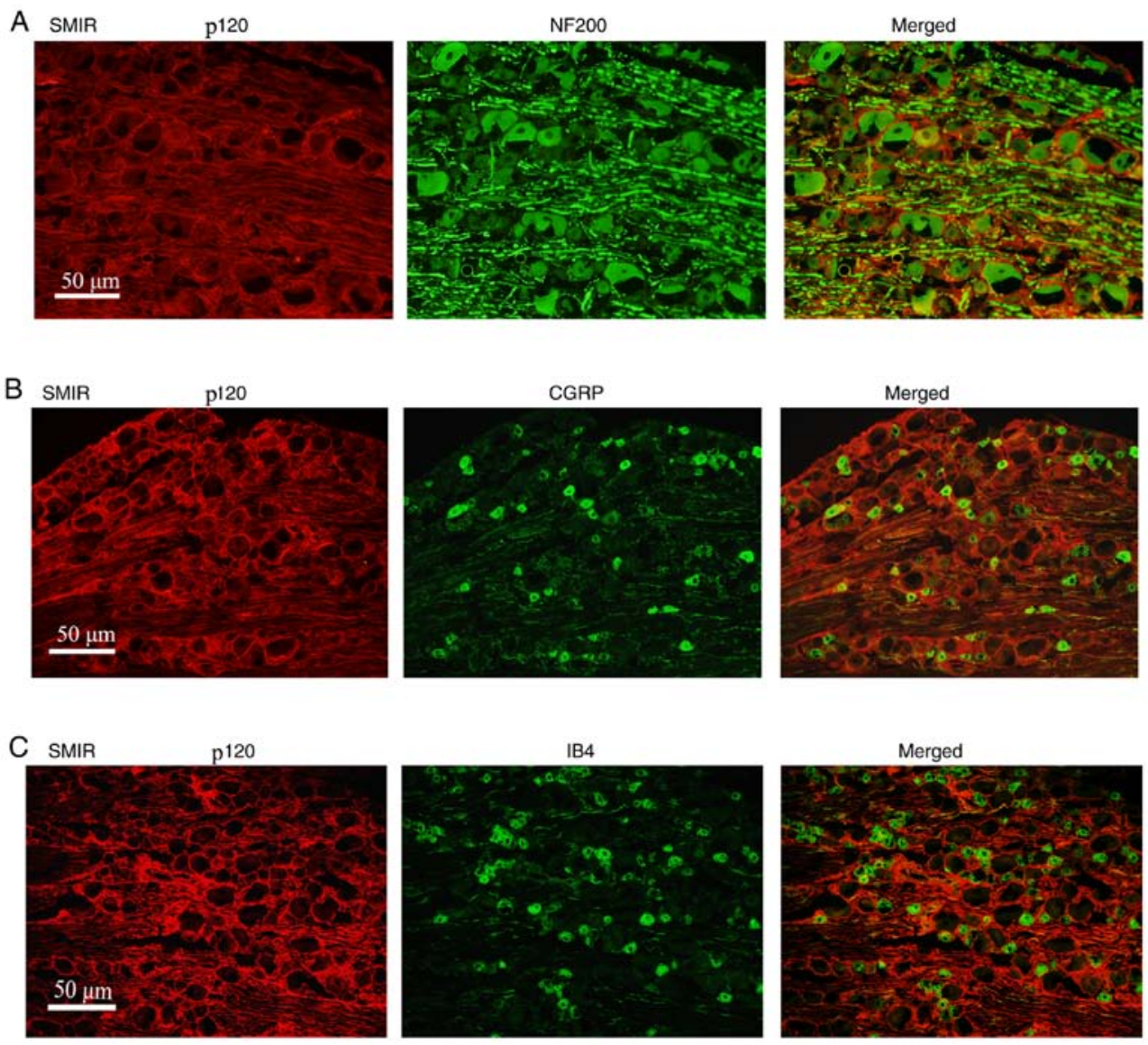

Figure 4. Immunofluorescence staining. Immunofluorescence staining demonstrated that in the DRG, p120 (A) colocalizes with the NF200-positive medium and large neurons, but does not colocalize with (B) CGRP- or (C) IB4-positive neurons. DRG, dorsal root ganglion, CGRP; calcitonin gene-related peptide; IB4, isolectin B4; SMIR, skin/muscle incision and retraction.
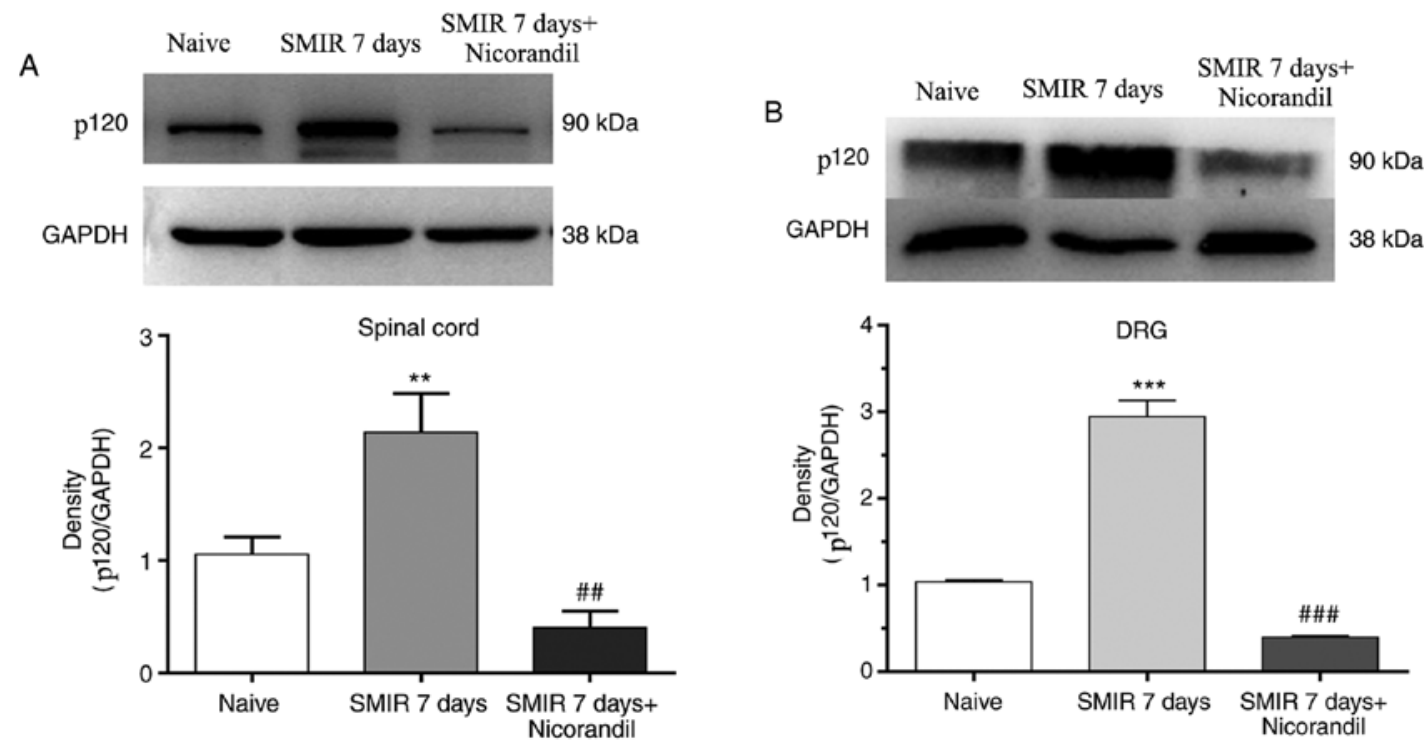

Figure 5. Expression of p120. (A) In the spinal cord, the SMIR group had significantly increased expression of p120 compared with in the naive group, and the SMIR $+1.5 \mathrm{mg} / \mathrm{kg}$ nicorandil group had significantly decreased p120 expression compared with in the SMIR group. (B) In the DRG, the expression of p120 was significantly increased in the SMIR group compared with in the naive group. Compared with in the SMIR group, the expression of p120 was significantly decreased in the SMIR $+1.5 \mathrm{mg} / \mathrm{kg}$ nicorandil group. ${ }^{* *} \mathrm{P}<0.01,{ }^{* * *} \mathrm{P}<0.001$ vs. naive group; ${ }^{\# \#} \mathrm{P}<0.01,{ }^{\# \# \#} \mathrm{P}<0.001$ vs. SMIR group. SMIR, skin/muscle incision and retraction; DRG, dorsal root ganglion.

It has been demonstrated that KATP channels serve critical roles in regulating membrane excitability and neurotransmitter release, and in providing neuroprotection $(37,38)$. Our previous studies demonstrated that KATP 
agonists can significantly reduce postoperative pain in rats by inhibiting the apoptosis of vascular endothelial cells around the incision $(10,11)$. The KATP activator nicorandil is a nitric oxide donor approved for the treatment of patients with angina pectoris, which also exhibits activities in models of inflammatory and nociceptive pain $(12,13)$. Regarding the cardiovascular actions of nicorandil, those that have been investigated more extensively, it has been concluded that this drug exhibits important differences when compared to the traditional organic nitrates, possibly due to its ability to open potassium channels (39). The present study provided an additional demonstration of the antinociceptive activity of nicorandil by demonstrating its activity in an SMIR model. It was identified that the antinociceptive effect induced by nicorandil was markedly attenuated by the KATP blocker glibenclamide, providing solid evidence of an important mechanism mediating the activity of nicorandil. In addition, the present study identified that nicorandil could decrease the expression of p120 in the DRG and spinal cord. Combined with the effects of nicorandil on the expression of p120, it was hypothesized that the antinociceptive activity of nicorandil may depend on the p120 pathway, thus expanding the scope of the study of the analgesic effects of nicorandil.

In summary, high expression of p120 may be the key link in central sensitization of CPSP. The regulation of p120 through targeted intervention by KATP agonists may be an effective strategy to inhibit central sensitization and may form the basis of potential therapeutic approaches for the treatment of CPSP.

\section{Acknowledgements}

Not applicable.

\section{Funding}

The present study was supported by the National Natural Science Foundation of China (grant no. 81701106).

\section{Availability of data and materials}

All data generated or analyzed during this study are included in this published article.

\section{Authors' contributions}

SSH and JPY designed the study. SSH, SC and CEL acquired and interpreted the data. YBQ analyzed the data and assisted JPY in revising the manuscript. SSH prepared the manuscript and supervised the study. All authors read and approved the final manuscript.

\section{Ethics approval and consent to participate}

All experiments in the current study were approved by the Experimental Animal Protection and Care Committee of the Nantong University (approval no. 20171015S1051122).

\section{Patient consent for publication}

Not applicable.

\section{Competing interests}

The authors declare that they have no competing interests.

\section{References}

1. Kim DH, Pearson-Chauhan KM, McCarthy RJ and Buvanendran A: Predictive factors for developing chronic pain after total knee arthroplasty. J Arthroplasty 33: 3372-3378, 2018.

2. Christensen RK, Delgado-Lezama R, Russo RE, Lind BL, Alcocer EL, Rath MF, Fabbiani G, Schmitt N, Lauritzen M, Petersen AV, et al: Spinal dorsal horn astrocytes release GABA in response to synaptic activation. J Physol 596: 4983-4994, 2018.

3. Wang S, Deng J, Fu H, Guo Z, Zhang L and Tang P: Astrocytes directly clear myelin debris through endocytosis pathways and followed by excessive gliosis after spinal cord injury. Biochem Biophys Res Commun: Feb 15, 2020 doi: 10.1016/j.bbrc.2020.02.069 (Epub ahead of print).

4. Nakagawa T and Kaneko S: Spinal astrocytes as herapeutic targets for pathological pain. J Pharmacol Sci 114: 347-353, 2010.

5. Xing L, Yang T, Cui S and Chen G: Connexin hemichannels in astrocytes: Role in CNS disorders. Front Mol Neurosci 12: 23, 2019.

6. Wang $\mathrm{A}$ and $\mathrm{Xu} \mathrm{C}$ : The role of connexin43 in neuropathic pain induced by spinal cord injury. Acta Biochim Biophys Sin (Shanghai) 51: 555-561, 2019.

7. Yang H, Yan H, Li X, Liu J, Cao S, Huang B, Huang D and Wu L: Inhibition of Connexin 43 and phosphorylated NR2B in spinal astrocytes attenuates bone cancer pain in mice. J Neurotrauma 12: 129, 2018.

8. Komiya H, Shimizu K, Ishii K, Kudo H, Okamura T, Kanno K, Shinoda M, Ogiso B and Iwata K: Connexin 43 expression in satellite glial cells contributes to ectopic tooth-pulp pain. J Oral Sci 60: 493-499, 2018.

9. Choi SR, Roh DH, Yoon SY, Kwon SG, Choi HS, Han HJ, Beitz AJ and Lee JH: Astrocyte sigma-1 receptors modulate connexin 43 expression leading to the induction of below-level mechanical allodynia in spinal cord injured mice. Neuropharmacology 111: 34-46, 2016.

10. Shen S, Cao S, Huang S and Chen J: Effect of adenosine triphosphate-sensitive potassium activation on peripheral and central pain sensitization. J Surg Res 195: 481-487, 2015.

11. Cao S, Qin Y, Chen J and Shen SR: Effects of pinacidil on changes to the microenvironment around the incision site, of a skin/muscle incision and retraction, in a rat model of postoperative pain. Mol Med Rep 12: 829-836, 2015

12. Dutra MM, Godin AM, César IC, Nascimento EB Jr, Menezes RR, Ferreira WC, Soares DG, Seniuk JG, Araújo DP, Bastos LF, et al: Activity of nicorandil, a nicotinamide derivative with a nitrate group, in the experimental model of pain induced by formaldehyde in mice. Pharmacol Biochem Behav 106: 85-90, 2013.

13. Dutra MM, Nascimento Júnior EB, Godin AM, Brito AM, Melo IS, Augusto PS, Rodrigues FF, Araújo DP, de Fátima Â, Coelho MM and Machado RR: Opioid pathways activation mediates the activity of nicorandil in experimental models of nociceptive and inflammatory pain. Eur J Pharmacol 768: 160-164, 2015.

14. Flatters SJ: Characterization of a model of persistent postoperative pain evoked by skin/muscle incision and retraction (SMIR). Pain 135: 119-130, 2008.

15. He WK, Su Q, Liang JB, Wang XT, Sun YH and Li L: Nicorandil pretreatment inhibits myocardial apoptosis and improves cardiac function after coronary microembolization in rats. J Geriatr Cardiol 15: 591-597, 2018.

16. Ahmed LA, Salem HA, Attia AS and Agha AM: Pharmacological preconditioning with nicorandil and pioglitazone attenuates myocardial ischemia/reperfusion injury in rats. Eur J Pharmacol 663: 51-58, 2011.

17. He W, Su Q, Liang J, Sun Y, Wang X and Li L: The protective effect of nicorandil on cardiomyocyte apoptosis after coronary microembolization by activating Nrf2/HO-1 signaling pathway in rats. Biochem Biophys Res Commun 496: 1296-1301, 2018.

18. Dixon WJ: Staircase bioassay: The up-and-down method. Neurosci Biobehav Rev 15: 47-50, 1991. 
19. Rhudy JL, Lannon EW, Kuhn BL, Palit S, Payne MF, Sturycz CA, Hellman N, Güereca YM, Toledo TA, Huber F, et al: Assessing peripheral fibers, pain sensitivity, central sensitization and descending inhibition in native Americans: Main findings from the oklahoma study of native American pain risk. Pain 161: 388-404, 2020.

20. Filbrich L, van den Broeke EN, Legrain V and Mouraux A: The focus of spatial attention during the induction of central sensitization can modulate the subsequent development of secondary hyperalgesia. Cortex 124: 193-203, 2019.

21. Ikeda H, Kiritoshi T and Murase K: Contribution of microglia and astrocytes to the central sensitization, inflammatory and neuropathic pain in the juvenile rat. Mol Pain 8: 43, 2012.

22. Gao YJ, Zhang L, Samad OA, Suter MR, Yasuhiko K, Xu ZZ, Park JY, Lind AL, Ma Q and Ji RR: JNK-induced MCP-1 production in spinal cord astrocytes contributes to central sensitization and neuropathic pain. J Neurosci 29: 4096-4108, 2009.

23. Zheng P, Jia S, Guo D, Chen S, Zhang W, Cheng A, Xie W, Sun G, Leng $\mathrm{J}$ and Lang J: Central sensitization-related changes in brain function activity in a rat endometriosis-associated pain model. J Pain Res 13: 95-107, 2020.

24. Mao Y, Tonkin RS, Nguyen T, O'Carroll SJ, Nicholson LF, Green CR, Moalem-Taylor G and Gorrie CA: Systemic administration of Connexin 43 mimetic peptide improves functional recovery after traumatic spinal cord injury in adult rats J Neurotrauma 34: 707-719, 2017.

25. Xu X, Li WE, Huang GY, Meyer R, Chen T, Luo Y, Thomas MP Radice GL and Lo CW: Modulation of mouse neural crest cell motility by $\mathrm{N}$-cadherin and connexin 43 gap junctions. J Cell Biol 154: 217-230, 2001.

26. Zhou L, Ao L, Yan Y, Li C, Li W, Ye A, Liu J, Hu Y, Fang W and $\mathrm{Li}$ Y: Levo-corydalmine attenuates vincristine-induced neuropathic pain in mice by upregulating the $\mathrm{Nrf} 2 / \mathrm{HO}-1 / \mathrm{CO}$ pathway to inhibit Connexin 43 expression. Neurotherapeutics 17: $340-355,2020$

27. Vicario N, Pasquinucci L, Spitale FM, Chiechio S, Turnaturi R, Caraci F, Tibullo D, Avola R, Gulino R, Parenti R and Parenti G: Simultaneous activation of mu and delta opioid receptors reduces allodynia and astrocytic Connexin 43 in an animal model of neuropathic pain. Mol Neurobiol 56: 7338-7354, 2019

28. Liu J and Huang D: Progress in gap junction protein 43 in chronic pain. Zhong Nan Da Xue Xue Bao Yi Xue Ban 43: 95-99, 2018 (In Chinese).
29. Zhang Y, Jiao H, Wu Y and Sun X: P120-catenin regulates pulmonary fibrosis and TGF- $\beta$ induced lung fibroblast differentiation. Life Sci 230: 35-44, 2019.

30. Garrett JP, Lowery AM, Adam AP, Kowalczyk AP and Vincent PA: Regulation of endothelial barrier function by $\mathrm{p} 120$ catenin-VE-cadherin interaction. Mol Biol Cell 28: 85-97, 2017.

31. Xie Z, Tang Y, Man MQ, Shrestha C and Bikle DD: p120-catenin is required for regulating epidermal proliferation, differentiation and barrier function. J Cell Physiol 234: 427-432, 2018.

32. Wehrendt DP, Carmona F, González Wusener AE, González Á, Martínez JM and Arregui CO: P120-catenin regulates early trafficking stages of the $\mathrm{N}$-cadherin precursor complex. PLoS One 11: e0156758, 2016.

33. Gritsenko PG, Atlasy N, Dieteren CEJ, Navis AC, Venhuizen JH, Veelken C, Schubert D, Acker-Palmer A, Westerman BA, Wurdinger T, et al: p120-catenin-dependent collective brain infiltration by glioma cell networks. Nat Cell Biol 22: 97-107, 2020.

34. Yuan L and Arikkath J: Functional roles of p120ctn family of proteins in central neurons. Semin Cell Dev Biol 69: 70-82, 2017.

35. Malcangio M: Role of the immune system in neuropathic pain. Scand J Pain 20: 33-37, 2019.

36. Chauvet N, Privat A and Prieto M: Differential expression of p120 catenin in glial cells of the adult rat brain. J Comp Neurol 479 15-29, 2004.

37. Roper J and Ashcroft FM: Metabolic inhibition and low internal ATP activate K-ATP channels in rat dopaminergic substantia nigra neurones. Pflügers Arch 430: 44-54, 1995.

38. Yamada K and Inagaki N: Neuroprotection by KATP channels. J Mol Cell Cardiol 38: 945-949, 2005

39. Ahmed LA: Nicorandil: A drug with ongoing benefits and different mechanisms in various diseased conditions. Indian J Pharmacol 51: 296-301, 2019.

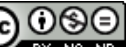

This work is licensed under a Creative Commons Attribution-NonCommercial-NoDerivatives 4.0 International (CC BY-NC-ND 4.0) License. 